\title{
Global Map Matching using BLE Beacons for Indoor Route and Stay Estimation
}

\author{
DAISUKE YAMAMOTO \\ Nagoya Institute of Technology \\ Nagoya, Aichi, Japan \\ daisuke@nitech.ac.jp
}

\author{
RYOSUKE TANAKA \\ Nagoya Institute of Technology \\ Nagoya, Aichi, Japan \\ ryosuke@moss.elcom.nitech.ac.jp
}

\author{
SHINSUKE KAJIOKA \\ Nagoya Institute of Technology \\ Nagoya, Aichi, Japan \\ kajioka@nitech.ac.jp
}

\author{
HIROSHI MATSUO \\ Nagoya Institute of Technology \\ Nagoya, Aichi, Japan \\ matsuo@nitech.ac.jp
}

\author{
NAOHISA TAKAHASHI \\ Nagoya Institute of Technology \\ Nagoya, Aichi, Japan \\ naohisa@nitech.ac.jp
}

\begin{abstract}
Recently, location service using Bluetooth Low-Energy (BLE) beacon is gaining popularity. There also exist researches that estimate the route of the user from the location estimation results, visualize, and analyze it. In the conventional route estimation method based on the BLE beacon, after estimating the all locations from the radio field strength of the BLE, the route is estimated from the sequence of the estimated locations. Therefore, one of the causes of deterioration in accuracy could be the fact that the estimation was repeated twice. Therefore, in this paper, we propose a novel estimation method of the user's route and stay using BLE beacons. Specifically, we apply the idea of global map matching, used in the field of GPS, to route estimation based on BLE beacons. Thus, the global optimum route in beacon network can be estimated from the radio field intensities of the BLE beacon directly. We associate a beacon network with path network to estimate the route and stay of users. We installed approximately 1,600 BLE beacon transmitters in all the classrooms and corridors of Nagoya Institute of Technology, and confirmed the effectiveness of the proposed method by experiments.
\end{abstract}

\section{CCS CONCEPTS}

- Information systems $\rightarrow$ Geographic information systems;

\section{KEYWORDS}

BLE Beacon, Map-matching, localization, trajectory, indoor navigation

\section{ACM Reference Format:}

DAISUKE YAMAMOTO, RYOSUKE TANAKA, SHINSUKE KAJIOKA, HIROSHI MATSUO, and NAOHISA TAKAHASHI. 2018. Global Map Matching using BLE Beacons for Indoor Route and Stay Estimation. In 26th ACM SIGSPATIAL International Conference on Advances in Geographic Information Systems (SIGSPATIAL '18), November 6-9, 2018, Seattle, WA, USA. ACM, New York, NY, USA, 10 pages. https://doi.org/10.1145/3274895.3274918

Permission to make digital or hard copies of part or all of this work for personal or classroom use is granted without fee provided that copies are not made or distributed for profit or commercial advantage and that copies bear this notice and the full citation on the first page. Copyrights for third-party components of this work must be honored. For all other uses, contact the owner/author(s).

SIGSPATIAL '18, November 6-9, 2018, Seattle, WA, USA

(C) 2018 Copyright held by the owner/author(s).

ACM ISBN 978-1-4503-5889-7/18/11.

https://doi.org/10.1145/3274895.3274918

\section{INTRODUCTION}

In recent years, the number of smartphones and devices equipped with Bluetooth Low Energy (BLE) beacon function has been on an increase. Consequently, many services using BLE beacons to estimate the location of users have been proposed. BLE beacons have broad applications such as guidance in public spaces, including universities [9] and museums [4, 10], and assistance for blind people [1]. We have also developed an attendance management system [14] using approximately 1600 beacons in all classrooms and corridors of the Nagoya Institute of Technology. This system allows students to report attendance through a smartphone application by accepting radio waves of the BLE beacons.

BLE is a Bluetooth standard that has been very efficient in reducing power consumption and allowing beacon devices to operate for several years on one battery. BLE beacon is compact, energy-efficient, inexpensive, easy to install, and can communicate with a smartphone. Because of such features, beacons are widely used for indoor location estimation studies.

BLE has a property in which the received signal strength indication (RSSI) is correlated to the distance between the transmitter and the receiver, which is theoretically defined by the conversion function. However, because BLE emits radio waves in the $2.4 \mathrm{GHz}$ band, there is a multipath problem characterized by the reflection and interference of radio waves due to the walls and floors of a building. In real environments, the further the distance from the BLE beacon, the lower the reliability of RSSI $[7,15]$. Therefore, to realize high-accuracy location estimation, it is necessary to install more BLE beacons.

On the other hand, in the field of GPS, many studies on road matching have been proposed. Road matching is a method of estimating a travel route in a road network based on the position series obtained from GPS. For example, the global map matching method $[3,16,17,20]$ can realize high-estimation accuracy even if the positional interval of GPS is rough. Although you may think global map matching can be easily applied to BLE beacons, because the principles of BLE beacons and GPS are very different, global map matching cannot be applied as it is. In addition, GPS can easily and mathematically estimate a position if four or more satellites are visible, but BLE cannot easily determine the position because of the multipath problem. Because location estimation is the main 


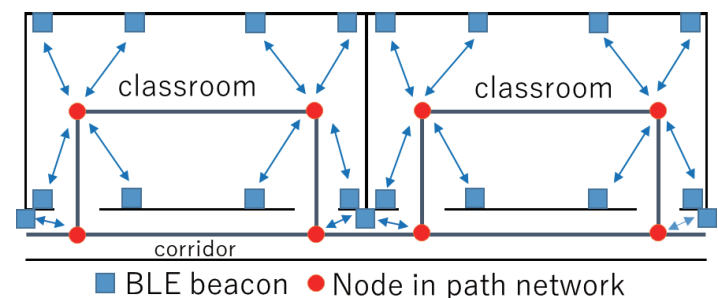

Figure 1: When the BLE beacon installation position does not match the node position of the path network

research topic in the field of BLE, therefore unlike GPS, there is little research on route estimation using BLE.

The purpose of this study is to realize a highly accurate indoor route / stay estimation of users by proposing a novel approach that implements global map matching techniques for route estimation using BLE beacons. Route estimation in this research refers to the estimation of a travel route in a path network composed of nodes and links. Stay estimation refers to the estimation of the time (start time and end time) staying at the node in a path network. The feature of this research is to realize a global optimal route estimation by making improvements to global map matching for GPS without using existing location estimation methods such as BLE beacons .

In realizing this method, the following problems can be mentioned.

Problem 1 Because the principles of BLE beacon and GPS are different, it is difficult to directly apply the global map matching method for GPS directly to the BLE beacon. For example, a GPS log is recorded as a series of latitude and longitude coordinates, but a BLE beacon log is recorded as a sequence of beacon ID and RSSI. In other words, the BLE beacon log does not contain position information.

Problem 2 Because the BLE beacon has a multipath problem in which reflection of radio waves occurs due to the walls and floors of buildings, the value of RSSI tends to be extremely unstable as the distance from the BLE beacon increases.

Problem 3 BLE beacons might not be placed directly above the nodes of the path network because of restrictions involving the location where the BLE beacons are installed. For example, as shown in Figure 1, the installation position of the BLE beacon and the position of the node on the path network may be at a certain distance from each other. Furthermore, in our university, to ensure beacon redundancy, multiple BLE beacons are installed at same location. Therefore, we have to merge multiple beacons to one beacon.

We will attempt to solve these problems using the following approaches.

Approach 1 We propose a BST-Matching method to realize global map matching using BLE beacons. This method is a novel technique based on ST-Matching, which is one of the global map matching methods for GPS.
Approach 2 We propose a method that preferentially uses beacons with large RSSI as described below. 1) When BLE beacon data with the same ID are consecutively recorded, they are integrated into one beacon data having the largest RSSI value. 2) To avoid the detection of BLE beacons with low reliability, filtering by threshold is performed. 2) In order not to detect BLE beacons with low reliability, filtering by threshold is performed.

Approach 3 We construct a two-layer network. First is the beacon network that represents the actual installation relationship of the BLE beacons, and the second is the path network that represents the route that users follow. To estimate the user's route / stay place in the path network, it converts from the route obtained by BST-Matching on the beacon network to the route on the path network.

The structure of this paper is as follows. We describe related research in Section 2. Section 3 describes the proposed method, and Section 4 describes the prototype system of the proposed method. Section 5 includes the experiment and discussion, and Section 6 provides the summary.

\section{RELATED WORK}

Incremental map matching [3] and global map matching [3, 16, $17,20]$ have been proposed as methods of route estimation from GPS point series data. Incremental map matching is a method of incrementally determining locally optimal paths on the road network from coordinates in the GPS log. Global map matching is a method of finding the global optimum route from the GPS point series data. Although global map matching tends to provide higher accuracy, there is also the problem of high calculation cost. Lou et al. [16] proposes ST-Matching, which can perform matching with high accuracy even when the GPS reception interval is rough. Considering spatial and temporal features, they derive the path using the Viterbi algorithm based on the score between each candidate point. White et al. [20] used curve-to-curve matching. Newson et al. [17] proposed map matching using the Hidden Markov Model.

In addition, research on location estimation using BLE beacons also include the proximity method [15], particle filtering method $[6,23]$, trilateration [2], and fingerprint method $[8,13]$. The proximity method is a simple method that involves setting a position where the beacon with the highest RSSI is the estimated position when radio waves from the beacons are received. The particle filter method is a method of deriving stochastic likelihood results based on the concept of Bayesian filter. Kajioka et al. [13] proposed position estimation using the fingerprint method. In the fingerprint method, an estimation model is constructed based on the beacon ID and RSSI measured for each point to be estimated in advance. The fingerprint method involves estimating a position from the beacon ID and RSSI and the estimated model observed when performing position estimation. Although a general location estimation method involves the installation of the BLE beacon in the environment and users have the BLE scanner, Urano et al. [19] proposed a method in which users have the BLE beacon and the BLE scanners are installed in the environment.

Research on location estimation using the radio waves of a Wifi router $[11,12,21]$ is also available. The wave frequency of the Wifi 


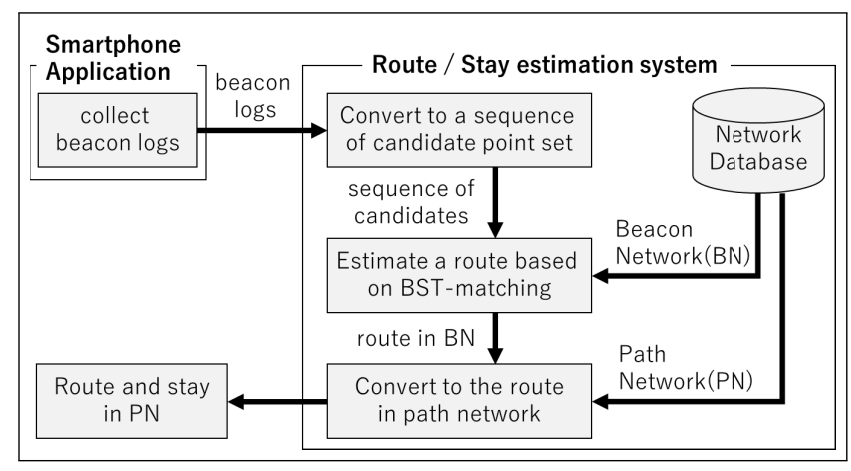

Figure 2: Outline of the proposed method

routers is the same as that of the BLE beacon, but the radio wave reaching distance is longer. Since Wifi routers is are not suitable for high high-density installations like a BLE beacon, it is necessary to estimate the location with a small number of wifi Wifi routers. In addition, Shorkry et al. [22] proposes proposed a mechanism for location estimation that combines a Wifi router and a BLE beacon.

Contrary to importance, many methods of route estimation using map matching have not been proposed. Stange et al. [18] proposed an analytical workflow for monitoring human mobility in big event settings using Bluetooth. Since this method estimates the route in free space, it is not map matching. There is also research on map-aided location estimation. Davidson et al. [5] stated that there are studies such as map-matching using wall constraints and topological map-matching for indoor navigation. For example, Dickinson et al. [6] proposed a location estimation method using a node graph and particle filter. The search space can be restricted by using the node graph, and the accuracy of location estimation is improved. However, map-aided location estimation is basically a location estimation method. Although it is possible to estimate the route by connecting the sequence of the results of location estimation, it is not a route estimation method that uses global map matching like the proposed method.

\section{PROPOSED METHOD}

An outline of the proposed method is shown in Figure 2. In the proposed method, a beacon network, representing the positional relationship of BLE beacons, and a path network, representing the route used by users, are constructed by using the network construction system described in Section 4.1. The proposed method then performs route / stay estimation based on the beacon log collected by a smartphone and the above-mentioned networks. The path on a beacon network is estimated by BST-Matching, proposed in this research, using the beacon network and beacon log. Next, proposed system converts the path on the beacon network into the path on the path network. Stay estimation is also possible based on the estimated route and the time recorded in the beacon log.

\subsection{Building Networks}

In this research, we propose a two layer network: beacon network and path network as shown in Figure 3. The beacon network is a graph based on the positional relationship of BLE beacons. The

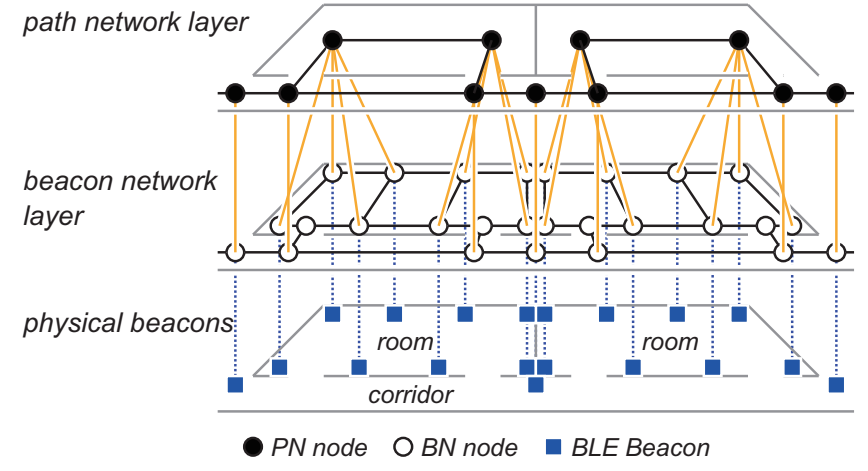

Figure 3: Model of two layer network : beacon network and path network for physical beacons.

Table 1: PN node table

\begin{tabular}{ccl}
\hline column name & data type & description \\
\hline node_id & int & PN node ID \\
type & int & node type \\
beacon_id & int[] & beacon ID \\
\hline
\end{tabular}

Table 2: PN link table

\begin{tabular}{ccl}
\hline column name & data type & description \\
\hline link_id & int & PN link ID \\
nd1 & int & start PN nodeID \\
nd2 & int & end PN node ID \\
cost & double & disitance between nd1 and nd2 \\
\hline
\end{tabular}

path network is a graph for defining the paths in which users move. The reason for constructing a beacon network is to realize BSTMatching, which is described at a later section.

3.1.1 Path Network $(P N)$. The path network is a graph that depicts the route in which users actually move. It is assumed that nodes are placed at important points as a route / stay place, including corridors, in front of doorways of rooms, in rooms, etc. The nodes of the path network are categorized according to the type of installation point, and there are 3 nodes: PN node (room), PN node (corridor), and PN node(connection).

Let the node on the path network be the PN node and the link be the PN link. The data structure of the PN node is shown in Table 1, and the data structure of the PN link is shown in Table 2. In addition, an example of a path network of the classroom environment in Figure 9 is shown in Figure 4.

3.1.2 Beacon Network(BN). A beacon network is a graph that depicts the beacon placement relations. Let the node on the beacon network be the $\mathrm{BN}$ node and the link be the $\mathrm{BN}$ link. The BN node is placed at the actual installation location of the beacon. However, the BN node is placed not only at the installation point of the beacon but also at the connection node on the path network, 


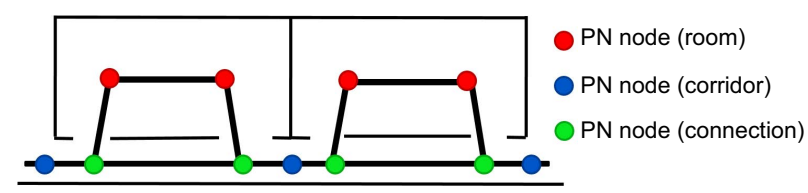

Figure 4: Example of path network

Table 3: Data structure of BN node

\begin{tabular}{ccl}
\hline column name & data type & description \\
\hline node_id & int & BN node ID \\
type & int & node type \\
beacon_id & int & beacon ID \\
\hline
\end{tabular}

Table 4: Data structure of BN link

\begin{tabular}{ccl}
\hline column name & data type & description \\
\hline link_id & int & BN link ID \\
nd1 & int & start BN node ID \\
nd2 & int & end BN node ID \\
cost & double & distance between nd1 and nd2 \\
\hline
\end{tabular}

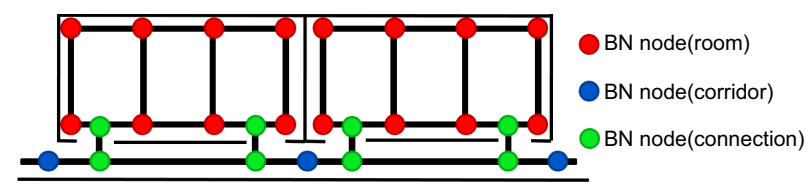

Figure 5: Example of a beacon network

including the entrance and exit of the room. The connection node on the beacon network is the same as the connection node on the path network, and the BN node arranged at the installation location of the beacon is defined as a beacon node. In addition, because the reflection characteristics of radio waves vary depending on the installation location of the beacon, we distinguish the beacon node between the room beacon and the corridor beacon. The BN node and the PN node have correspondence via the beacon ID.

The data structure of the $\mathrm{BN}$ node is shown in Table 3, and the data structure of the BN link is shown in Table 4. Node types of the $\mathrm{BN}$ node include 0 : $\mathrm{BN}$ node (connection), 1 : BN node (room), and 2: BN node (corridor). An example of the beacon network in Figure 9 is shown in Figure 5.

\subsection{Route and stay estimation method}

Next, we describe the route and stay estimation methods using a beacon network, a path network, and beacon logs.

3.2.1 Beacon log. The beacon log in this research is defined as a sequence in which beacon messages are recorded in chronological order. The beacon message contains the time of observation of the beacon and 0 or more beacon data (beacon ID and RSSI) recognized at that time. In the beacon message, the beacon data is repeatedly
Table 5: Data structure of candiate point

\begin{tabular}{cc}
\hline column name & structure \\
\hline BN node ID & Beacon Node ID \\
Beacon ID & Beacon ID \\
RSSI & Observed RSSI \\
time & Observed time \\
\hline
\end{tabular}

acquired during the scanning time ( $m$ seconds) with the observation frequency ( $n$ seconds). The beacon ID is defined by a Major ID, and the RSSI is a numerical value of the radio field intensity emitted by the beacon. In this research, the scan time $m$ was set to $1 \mathrm{~s}$ and the observation frequency $n$ was set to $1 \mathrm{~s}$.

3.2.2 Integration and filtering of beacon message. To solve the problem in which the RSSI tends to become unstable as described in Problem 2, we integrate and filter the beacon message every certain number .

First, the procedure of integrating the beacon message is shown below.

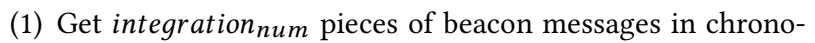
logical order from the beacon log.

(2) The acquired beacon messages are integrated as one beacon message in the following procedure. If multiple beacon data have the same beacon ID, the RSSI of the beacon data is assumed to be the largest value among them. Otherwise, do not change the contents of the beacon data.

Next, we describe the filtering of the beacon message. Filtering is achieved by removing beacon data below the threshold value $R$. Here, the threshold value $R$ of the room node can be different from the threshold value of the corridor node.

3.2.3 Generation of the sequence of candidate point set. We describe a method of converting the beacon message mentioned in the previous subsection into input data for the proposed BST-Matching method. In BST-Matching, we need to convert a beacon message into a sequence of candidate point sets as shown in Table 5 to estimate the route on the beacon network. In particular, the following processing is performed on the beacon message.

(1) Get the top $k$ beacon data (beacon ID, RSSI) with large RSSI value from the beacon message at time $i$.

(2) Get the BN node IDs corresponding to the candidate beacon set by referring to Table 3 .

(3) Generate K number of candidate points having BN node ID, beacon ID, RSSI, and time $i$ obtained in (1) and (2). The structure of the candidate point is shown in Table 5.

As a result, a candidate point set $\left(c_{i}^{1}, c_{i}^{2}, \ldots, c_{i}^{k}\right)$ can be generated for each time $i$ and that sequence becomes a sequence of candidate point sets.

3.2.4 Route generation for GPS by ST-Matching. We first outline the algorithm of ST-Matching [16] proposed by Lou et al.

In ST-Matching, the series of GPS data (latitude and longitude) $p_{1}, \ldots, p_{n}$ and the road network $G=(V, E)$ serve as inputs. At each time $i$, we acquire a set of vertices (candidate points) $c_{i}^{t}$ on the road network with radius $r$ meter from the latitude and longitude 


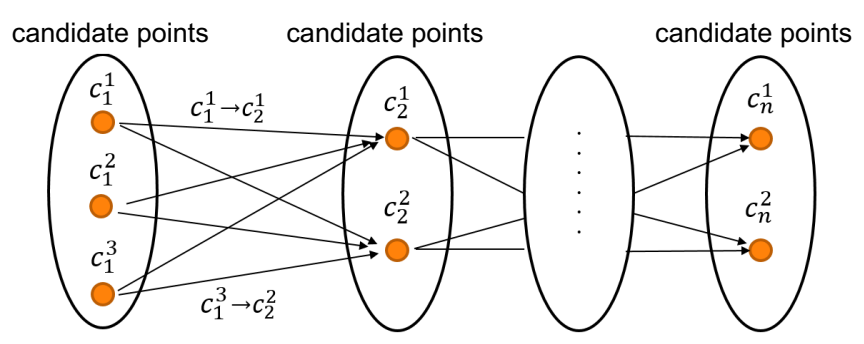

Figure 6: Candidate graph $G_{T}^{\prime}\left(V_{T}^{\prime}, E_{T}^{\prime}\right)$

readings in the GPS data. Repeat this process for all input times and construct the candidate graph $G_{T}^{\prime}$ shown in Figure 6. Calculate the evaluation formula $F\left(c_{i-1}^{t} \rightarrow c_{i}^{s}\right)$ for each edge of the candidate graph. The path with the smallest evaluation expression is then determined by the Viterbi algorithm.

Here, the evaluation formula $F\left(c_{i-1}^{t} \rightarrow c_{i}^{s}\right)$ is defined by the following expression.

$$
\begin{gathered}
F\left(c_{i-1}^{t} \rightarrow c_{i}^{s}\right)=F_{s}\left(c_{i-1}^{t} \rightarrow c_{i}^{s}\right) F_{t}\left(c_{i-1}^{t} \rightarrow c_{i}^{s}\right) \\
F_{s}\left(c_{i-1}^{t} \rightarrow c_{i}^{s}\right)=N\left(c_{i}^{s}\right) V\left(c_{i-1}^{t} \rightarrow c_{i}^{s}\right)
\end{gathered}
$$

Here, overviews of $F_{t}\left(c_{i-1}^{t} \rightarrow c_{i}^{s}\right), N\left(c_{i}^{s}\right), V\left(c_{i-1}^{t} \rightarrow c_{i}^{s}\right)$ for STMatching are defined as follow.

(1) $N\left(c_{i}^{j}\right)$ represents the proximity of the distance between the candidate point and the observation point. In other words, the score is determined based on the proximity of the observation point to the road network.

(2) $V\left(c_{i-1}^{t} \rightarrow c_{i}^{s}\right)$ represents the ratio between the distance on the path between the candidate points and the Euclidean distance between observation points. The closer the value is to 1 , the lesser is the likelihood of the user to detour.

(3) $F_{t}\left(c_{i-1}^{t} \rightarrow c_{i}^{s}\right)$ indicates whether the traveling speed on the route between the candidate points is close to the speed calculated from the route.

3.2.5 Route generation for BLE by BST-Matching. ST-Matching is an algorithm for GPS and road network, but BST-Matching is an algorithm for BLE beacons and beacon networks. We describe the BST-Matching algorithm while referring to ST-Matching.

(1) The set of candidate points for ST-Matching is the vertex set of the road network included in the radius $r$ of each GPS data point. In BST-Matching, the set of candidate points is a set of BN nodes corresponding to BLE beacons whose RSSI is larger than the threshold at each time point.

(2) Because the evaluation formula F of ST-Matching cannot be applied to the BLE beacon as it is, it is redefined at a later section. Like the ST matching, BST-matching defines the cost function $F^{\prime}\left(c_{i-1}^{t} \rightarrow c_{i}^{s}\right)$ using the spatial functions $N^{\prime}\left(c_{i}^{s}\right), V\left(c_{i-1}^{t} \rightarrow c_{i}^{s}\right)$ and the time function $F_{t}^{\prime}\left(c_{i-1}^{t} \rightarrow c_{i}^{s}\right)$.

Table 6 shows the difference between ST-Matching and the proposed method.

First, let's redefine candidate point $c_{i}^{s}$ for BST-matching. Candidate point $c_{i}^{s}$ is the sth candidate point at time $i$, described in
Section 3.2.3. Then the candidate graph $G_{T}^{\prime}$ is constructed by connecting adjacent candidate point set, as shown in Figure 6.

The score $N\left(c_{i}^{s}\right)$ is defined as follows, considering the RSSI.

$$
N^{\prime}\left(c_{i}^{s}\right)=\frac{1}{10^{\frac{1}{20}\left(R S S I_{\max }-R S S I\left(c_{i}^{s}\right)\right)}}
$$

However, RSSI $I_{\max }$ varies depending on the type of beacon installation location. In this study, we set the room beacon to -55 and the corridor beacon to be -60 .

Next, $F_{t}^{\prime}\left(c_{i-1}^{t} \rightarrow c_{i}^{s}\right)$ is defined as follows. $F_{t}^{\prime}\left(c_{i-1}^{t} \rightarrow c_{i}^{s}\right)$ is an evaluation expression for detecting unnatural moving speed by calculating the ratio of the assumed walking speed $v_{a}$ and the actual moving speed $v$ between candidate points $c_{i-1}^{t}, c_{i}^{s}$,

$$
\begin{gathered}
F_{t}^{\prime}\left(c_{i-1}^{t} \rightarrow c_{i}^{s}\right)= \begin{cases}1 & \left(v \leq v_{a}\right) \\
\frac{v_{a}}{v} & \left(v>v_{a}\right)\end{cases} \\
v=\frac{L\left(c_{i-1}^{t} \rightarrow c_{i}^{s}\right)}{T\left(c_{i-1}^{t} \rightarrow c_{i}^{s}\right)}
\end{gathered}
$$

Where, $L\left(c_{i-1}^{t} \rightarrow c_{i}^{s}\right)$ is the length of the shortest path between the BN nodes $\left(c_{i-1}^{t}, c_{i}^{s}\right)$ on the beacon network, and $T\left(c_{i-1}^{t} \rightarrow c_{i}^{s}\right)$ is the elapsed time between $c_{i-1}^{t}$ and $c_{i}^{s}$. In addition, $v_{a}$ was set as 2.0 $\mathrm{m} / \mathrm{s}$ in this study. The range of the score is $0<F_{t}^{\prime}\left(c_{i-1}^{t} \rightarrow c_{i}^{s}\right) \leq 1$, and the score approaches 0 faster than the average walking speed.

Finally, we define $V^{\prime}\left(c_{i-1}^{t} \rightarrow c_{i}^{s}\right)$. This function shows the ratio of the distance between the candidate points and the Euclidean distance between observation points. However, in the case of beacons, we can not directly obtain the observation point, and so we simply defined it as follows.

$$
V^{\prime}\left(c_{i-1}^{t} \rightarrow c_{i}^{s}\right)=1
$$

Using these evaluation expressions from (3) to (6), we can calculate the function (1) and (2) in the previous section as follows.

$$
\begin{gathered}
F^{\prime}\left(c_{i-1}^{t} \rightarrow c_{i}^{s}\right)=F_{s}^{\prime}\left(c_{i-1}^{t} \rightarrow c_{i}^{s}\right) F_{t}^{\prime}\left(c_{i-1}^{t} \rightarrow c_{i}^{s}\right) \\
F_{s}^{\prime}\left(c_{i-1}^{t} \rightarrow c_{i}^{s}\right)=N^{\prime}\left(c_{i}^{s}\right) V^{\prime}\left(c_{i-1}^{t} \rightarrow c_{i}^{s}\right)
\end{gathered}
$$

Therefore, we can estimate the route on the beacon network using the Viterbi algorithm, the candidate graph $G_{T}^{\prime}$ and evaluation formula $F^{\prime}\left(c_{i-1}^{t} \rightarrow c_{i}^{s}\right)$.

3.2.6 Conversion to the route on path network. We described a method by which BST-Matching generates a path on the beacon network from the beacon log. However, the beacon network is a model for the positional relationship of the beacons, which is different from the path network of the moving path of users. Therefore, referring to the relation between the beacon network and the path network, the proposed system converts to the route on the path network. However, the nodes of the converted route may not be connected to each other on the route. Therefore, nodes of a path network that are not connected can be connected based on Dijkstra's method. Complementing the nodes in this way makes it possible to acquire the routes connected on the path network. 
Table 6: Difference between ST-Matching and BST-Matching (proposed method)

\begin{tabular}{cll}
\hline & ST-matching & BST-matching \\
\hline input data & latitude and longitude & RSSI \\
candidate point & vertices within radius $r$ of GPS point & BLE beacons whose RSSI is larger than threshold $R$ \\
network & road network & beacon network \\
algorithm & Viterbi algorithm & Viterbi algorithm \\
\hline
\end{tabular}

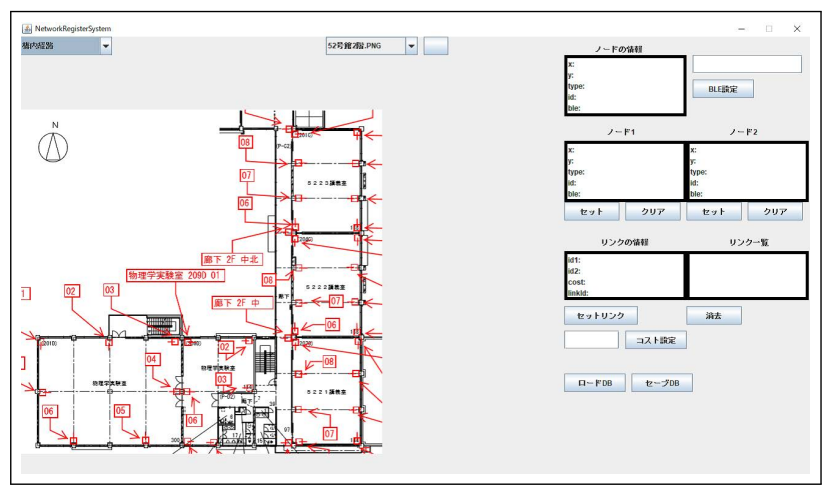

Figure 7: Screenshot of the network construction system

3.2.7 Stay estimation. Stay estimation is a function for estimating the place where a user stayed for a certain period of time and the period of stay. When the time of stay in the PN node on the path network exceeds the threshold value $T$ seconds, it is determined that the user stayed in the PN node. Here, when a certain PN node $X$ was continuously obtained $x$ times, it is judged that the user has stayed at the PN node $\mathrm{X}$ when the following expression is satisfied.

$$
n x \geq T
$$

Where, $n$ is the observation frequency of the BLE beacons.

\section{PROTOTYPE SYSTEM}

In this research, we implemented not only the proposed method but also a smartphone application that collects beacon logs, a network construction system, and a route / stay estimation result visualization system.

\subsection{Network construction system}

In the network construction system, it is possible to construct a path network and a beacon network as shown in Figure 7. PN nodes and $\mathrm{BN}$ nodes can be added by clicking on the map, and nodes can be associated with beacon IDs as inputs at the upper right corner of the screen. In addition, by selecting two nodes, it is possible to set PN link and BN link. It is also possible to set the type of node and the cost of the link.

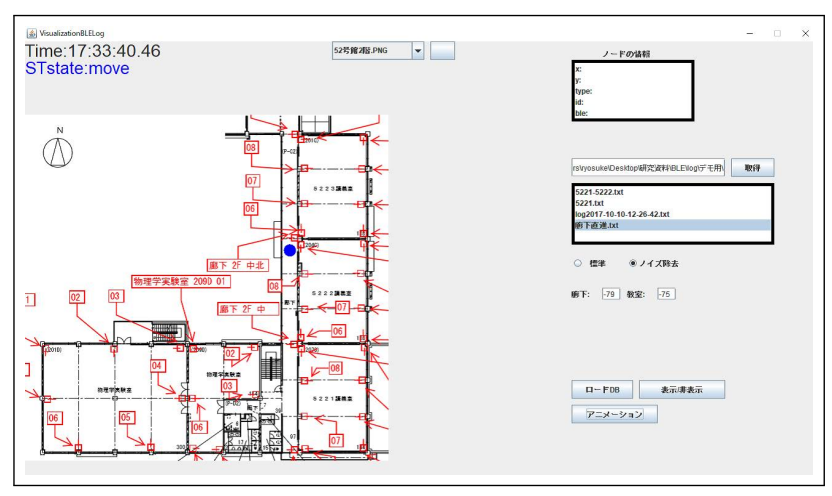

Figure 8: Example of animation: the user is moving

\subsection{Route and stay estimation result visualization system}

The route and stay estimation result visualization system has the same interface as the network construction system and can visualize the route / stay estimation result as shown in Figure 8. Path / stay estimation is performed by pressing the animation button after noise removal and threshold setting. Consequently, the estimation result is displayed in an animated form on the map.

\section{EXPERIMENTAL RESULT}

The purpose of the experiment is to evaluate whether the proposed method can perform estimation with high precision in route and stay estimation as compared with the conventional method. The conventional method is an incremental map matching method using a BLE beacon that will be described in a later section. In addition, we evaluate the proposed method by comparing the route / stay estimation result estimated by the prototype system with the true route.

\subsection{Incremental map matching using BLE beacon}

We propose a route estimation method using incremental map matching as a comparison target for the proposed method. In BST - Matching, as described in Section 3.2.3, multiple candidate points at time t were acquired, and the optimum route among the candidate points can be estimated. In incremental map matching, the number of candidate points at time $t$ is set to 1 . In other words, only the beacon having the largest RSSI is set as a candidate point. As a result, the route can be incrementally estimated by connecting the candidate points with the largest RSSI. Other conditions such as conversion 


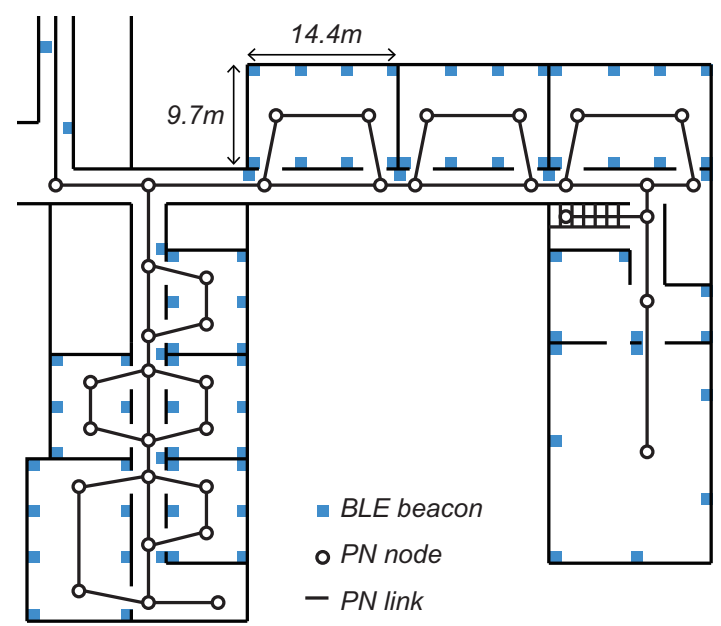

Figure 9: BLE beacon installation map and its path network

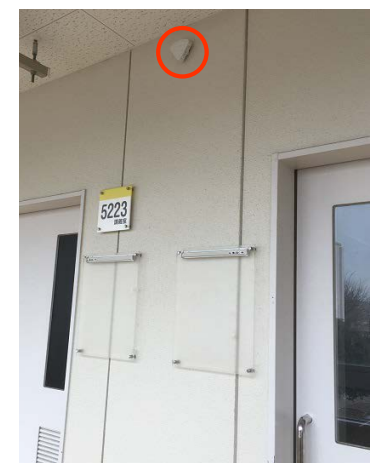

Figure 10: Installation example of BLE beacon (corridor). BLE beacon is in red circle

from beacon network to path network and filtering are the same as BST-Matching.

\subsection{Experiment environment}

In this research, we conducted an evaluation experiment using BLE beacons [14] installed for an attendance management system operated at the Nagoya Institute of Technology. Approximately 1,600 beacon transmitters were installed at all locations in the campus including all classrooms. The transmission interval of the beacon is $760 \mathrm{~ms}$. Figure 9 demonstrates an example of beacon installation installed on a certain floor of a certain building. In the classroom, 4 to 8 beacons are installed for communication stability and fault tolerance. Beacons are also installed in the hallway in front of the classroom as shown in Figure 10. A plurality of beacons are installed in all classrooms for communication stability and redundancy of the attendance management system, but the number of corridors is small and it is not possible to cover all locations. For example, beacons are not installed along stairways.

This experiment was conducted on the 2nd and 3rd floors of the common education buildings (Nos. 52 and 53) at the Nagoya
Table 7: Number of installed beacons

\begin{tabular}{cc}
\hline place & number of beacons \\
\hline Bld. 52, 2nd floor & 38 \\
Bld. 52, 3rd floor & 28 \\
Bld. 53, 2nd floor & 40 \\
\hline
\end{tabular}

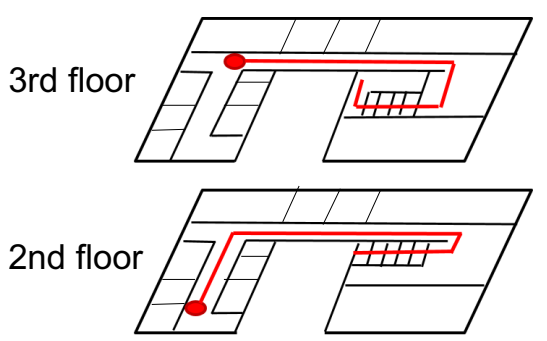

Figure 11: Route 1. It is consisting of stairs and corridors

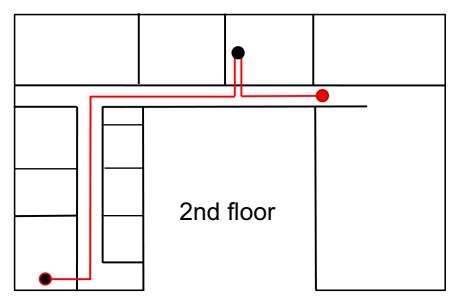

Figure 12: Route 2. Stop at a black circle.

Institute of Technology. The target area is about 2,500 square meters. We performed the experiment in the classroom and corridor in the absence of anyone. We constructed a beacon network and a path network based on the BLE beacon installed in the experiment environment beforehand and estimated routes and stays using these networks. The number of beacons in the target environment is shown in Table 7.

In addition, the walking route used in the experiment is Route 1, 2, 3 shown in Figure 11, Figure 12, Figure 13. Subjects used a smartphone with the beacon log collection application and moved along the routes 1, 2, and 3 on foot. The subjects moved again after stopping for a specified number of seconds at a given point (point of black circle). We collected 10 beacon logs for each route. In addition, by recording the time at each designated position, the grand true value of the time at the designated point was also recorded by clicking a button on the smartphone.

We set these routes based on the assumption of various use cases so that there is no bias in the experimental results. Route 1 assumes a route that passes only through corridors and stairs. Route 2 and route 3 set a route through both the classroom and the corridor. Route 2 and route 3 pass through the same classroom but use different entrances. Figures 14 to 17 show the average of the experimental results for each route.

5.2.1 Experiment. Experiment 1 and Experiment 2 described below were performed using the route and network described in the 


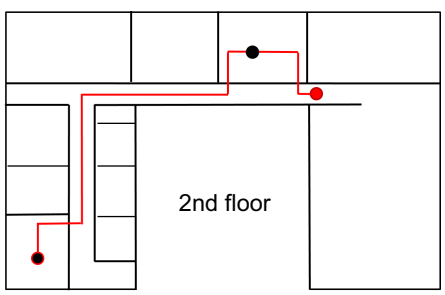

Figure 13: Route 3. Stop at a black circle

Table 8: Threshold for RSSI filtering

\begin{tabular}{cccccccccc}
\hline & 1 & 2 & 3 & 4 & 5 & 6 & 7 & 8 & 9 \\
\hline corridor & -83 & -81 & -79 & -77 & -75 & -73 & -71 & -69 & -67 \\
classroom & -81 & -78 & -75 & -72 & -69 & -66 & -63 & -60 & -57 \\
\hline
\end{tabular}

previous section. The methods used for estimation include the BSTMatching method, which is the proposed method, and the incremental map matching method, which is the comparison method.

Experiment 1 Experiment on route estimation. Change thresholds $R$ of RSSI as shown in Table 8 , and evaluate the accuracy in each method.

Experiment 2 Experiment on the estimation of staying time. In Routes 2 and 3, record the time of entering the classroom, the time of passing through the center, and the time of leaving the classroom, and compare the estimated time and the true value of each point.

Because it is expected that the experimental result will vary depending on the method of setting the threshold, in Experiment 2, the following four patterns are prepared according to the level of the threshold.

BST-L BST-Matching(Proposed method, Threshold:Low)

BST-H BST-Matching(Proposed method, Threshold:High)

Inc-L Incremental Map Matching(Threshold:Low)

Inc-H Incremental Map Matching(Threshold:High)

Where, when threshold is low, (corridor, room $)=(-79,-75)$. When threshold is high, (corridor, room $)=(-71,-63)$.

\subsection{Experimental Result}

The results of experiment 1 are shown in Figure 14, Figure 15, and Figure 16. Figure 14 shows the precision, Figure 15 shows the recall, and Figure 16 shows the F-measure of route estimation for each threshold, as listed in Table 8. In addition, we performed t-test between BST-matching and Incremental Map Matching for each threshold. * shows the sifnificant difference at a significace level of 0.05 . The precision, recall, and F-measure were calculated using the following formula:

L1 a set of links on the path network representing the estimated route.

L2 a set of links on the path network representing the route actually walked (correct route)

Precision $P=|L 1 \cap L 2| /|L 1|$

Recall $R=|L 1 \cap L 2| /|L 2|$
F-measure $F=2 P R /(P+R)$

where $|x|$ is the number of elements in set $x$, and $x \cap y$ is the intersection of $x$ and $y$. The ground truth is a link sequence on the path network showing the routes displayed in Figures 11 to 13. Figure 9 shows the structure of links on the path network.

As shown in Figure 14, both the incremental map matching and the BST-Matching improves the precision of route estimation as the threshold increases. As the threshold value is increased, candidate points with low RSSI value are removed, suggesting that beacons with low RSSI value have an adverse effect on the precision of route estimation. On the contrary, as shown in Figure 15, when the threshold value is set too large, the recall of the route estimation is extremely low. Figures 17 show the number of candidate nodes in the estimated path for each threshold. When the threshold value is increased, the number of nodes decreases. These results suggest that extremely decreasing candidate points as compared to the estimated path lead to a decrease in recall ratio. Consequently, as shown in Figure 16, the F-measure was a graph with the threshold $(-73,-66)$ as the peak. In other words, the larger the threshold value, the better is the estimation accuracy. In contrast, if the threshold value is too large, the accuracy deteriorates extremely.

The incremental map matching is then compared with BST-Matching. As shown in Figure 16, there was only a little difference in F-measure between BST-matching and Incremental map matching at the best thresholds $(-71,-63)-(-69,-60)$. However, in the area where the threshold is low, the accuracy (F-measure) of BST-Matching is improved, which suggests that BST-Matching is less sensitive to threshold than incremental map matching. As shown in Table 9, the Fmeasure of BST-Matching provided a good value of 0.9466 at the maximum. Furthermore, the range of F-measure (0.055) was $42 \%$ better than that of incremental map matching (0.095). In general, the RSSI of the BLE beacon is easily varied because of the voltage of the battery or the crowd, and it is difficult to set an optimum threshold value in a real environment. Therefore, it is desirable that accuracy be insensitive to the threshold value. Therefore, the proposed method is an excellent method, especially in the real environment.

Generally, it is not easy to find the best threshold value because the Received Signal Strength Indication (RSSI) of Bluetooth tends to change according to the crowd, humidity, and remaining battery power. Even if the best threshold can be set, this value will change according to these external factors. We must, therefore, find the optimal and stable threshold rather than the best threshold.

The proposed method is robust against changing the threshold. For example, as shown in Figure 16, both the proposed method (BST) and the comparison method (Inc) obtain best results when the threshold set is $(-69,-60)$. Here, paying attention to the threshold value (-79.-75), which is $10 \mathrm{~dB}$ lower than the best threshold value, the F-measure of the proposed method is much better than that of the comparison method. That is to say, the proposed method maintains good results even if the threshold is lowered by $10 \mathrm{~dB}$ from the best threshold value. On the other hand, when the threshold value is increased from the best value, both methods experience a problem in that the F-measure drops dramatically.

The optimal threshold is, therefore, decided based on the results of the preliminary experiment as follows. If the best threshold is 


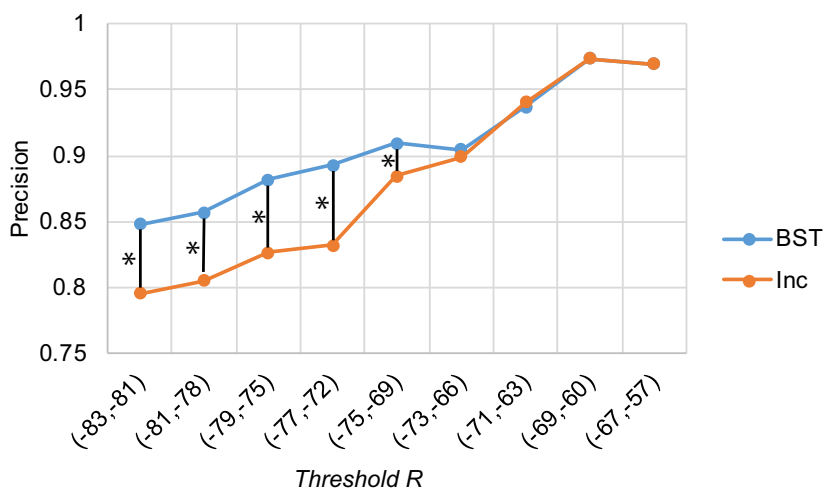

Figure 14: Precision of route estimation in Experiment 1. * shows the difference at a significance level of 0.05

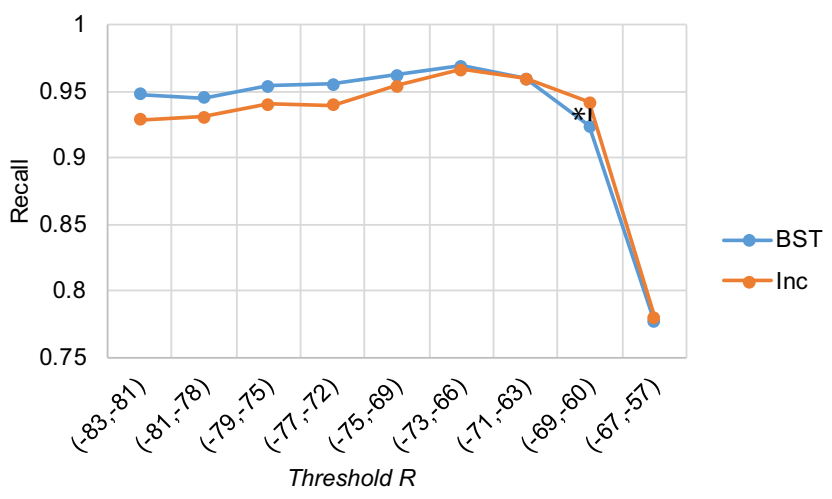

Figure 15: Recall of route estimation in Experiment 1. * shows the difference at a significance level of 0.05

Table 9: Detail of results in Experiment 1

\begin{tabular}{ccccccc}
\hline & \multicolumn{3}{c}{ BST-Matching } & \multicolumn{3}{c}{ Incremental map matching } \\
& Prec. & Rec. & FMeas. & Prec. & Rec. & FMeas. \\
\hline Max & 0.9373 & 0.9692 & 0.9466 & 0.9406 & 0.9665 & 0.9484 \\
Min & 0.8480 & 0.9448 & 0.8914 & 0.7958 & 0.9287 & 0.8530 \\
Range & 0.0893 & 0.0243 & 0.0552 & 0.1449 & 0.0378 & 0.0953 \\
\hline
\end{tabular}

$\mathrm{X} \mathrm{dB}$ and the range of RSSI changing is $\pm \mathrm{Y} \mathrm{dB}$ as determined by preliminary experiment, the optimal threshold is $(\mathrm{X}-\mathrm{Y}) \mathrm{dB}$. For example, when the best threshold is $-69 \mathrm{~dB}$ and the range of RSSI changing is $\pm 5 \mathrm{~dB}$, the optimum threshold is $-74 \mathrm{~dB}$. As a result, we can expect that the estimation result will be both stable and good, even if the RSSI changes in the range of $\pm 5 \mathrm{~dB}$. Here, since we use different threshold values in the corridor and the room, we have to calculate the optimum threshold for each area.

Next, the estimation result of the stay time in Experiment 2 is shown in Tables 10 and 11. In both methods, the lower the threshold, the smaller the error. Because the beacon can be detected with high sensitivity if the threshold is low, the staying time can be more

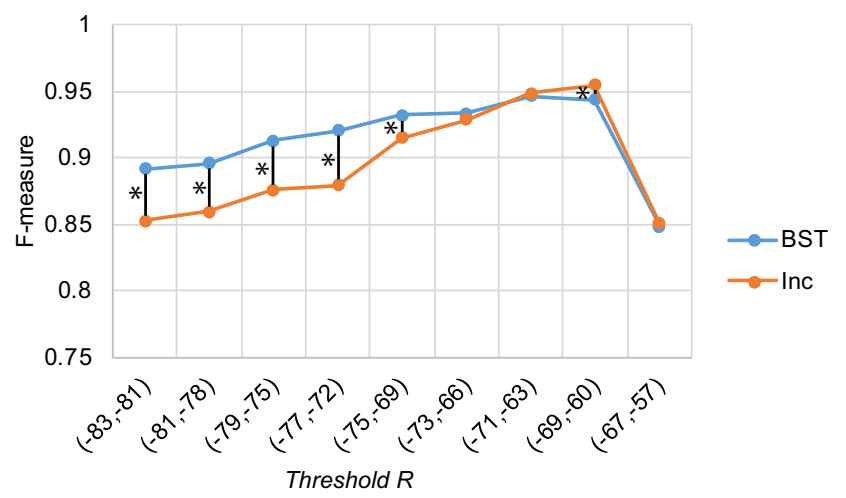

Figure 16: F-measure of route estimation in Experiment 1. * shows the difference at a significance level of 0.05

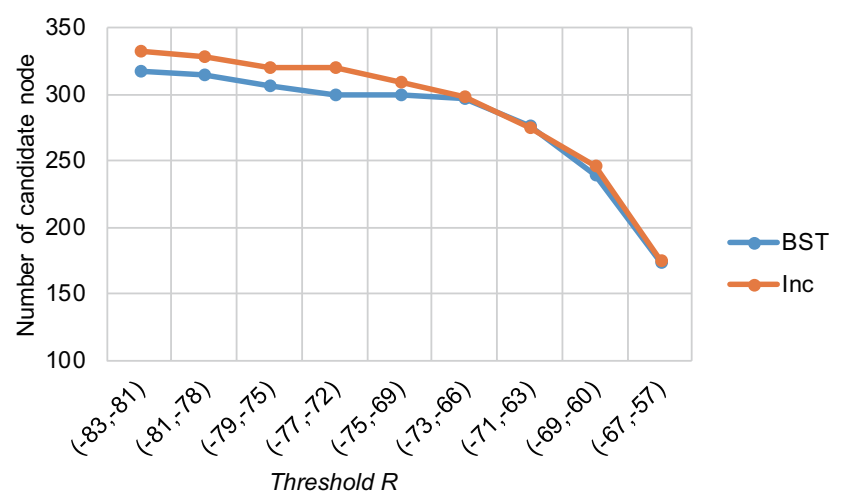

Figure 17: The number of candidate nodes in Experiment 1

Table 10: The result of stay estimation at Route 2 in Experiement 2

\begin{tabular}{cccc}
\hline method & error(sec) & standard deviation & correct rate \\
\hline BST-L & 4.5170 & 2.0400 & 1.000 \\
Inc-L & 6.3810 & 2.6668 & 1.000 \\
BST-H & 8.7250 & 5.2690 & 0.950 \\
Inc-H & 8.4250 & 5.5656 & 0.950 \\
\hline
\end{tabular}

accurately determined. When the threshold was low, the BST-Matching was up to $29 \%$ better than the incremental map matching. These results suggest that the BST-L method is the best for accuracy of stay estimation.

Finally, the average processing time of each method is shown in Table 12. In incremental map matching, the processing time is fast, while BST-Matching takes time because it is necessary to calculate the optimal solution between the candidate points. Particularly, when the threshold value is small, there is a tendency for the number of candidate points to increase, which consumes more processing time. Future work would involve improving processing time in BST-Matching. 
Table 11: The result of stay estimation at Route 3 in Experiement 2

\begin{tabular}{cccc}
\hline method & error(sec) & standard deviation & correct rate \\
\hline BST-L & 15.679 & 9.0095 & 0.950 \\
Inc-L & 21.239 & 9.7209 & 0.925 \\
BST-H & 27.317 & 12.622 & 0.825 \\
Inc-H & 26.925 & 11.448 & 0.825 \\
\hline
\end{tabular}

Table 12: Processing time (millisecond)

\begin{tabular}{lcccc}
\hline & BST-L & Inc-L & BST-H & Inc-H \\
\hline Route 1 & 5192.3 & 21.6 & 1384.7 & 74.6 \\
Route 2 & 8429.4 & 20.9 & 2102.5 & 105.6 \\
Route 3 & 9777.6 & 19.8 & 2554 & 103.6 \\
\hline
\end{tabular}

\section{CONCLUSION}

In this paper, we propose a novel estimation method of the user's route and stay using BLE beacons. Specifically, we apply the idea of global map matching, used in the field of GPS, for route and stay estimation based on BLE beacons. Therefore, the global optimum route in a beacon network can be directly estimated from the RSSI of the BLE beacon by using the Viterbi algorithm. Moreover, we proposed a two layer network method (path network and beacon network) and an integration / filtering method of beacon data to estimate the route with high accuracy in real environments.

The evaluation experiment demonstrated that the proposed method has very good F-measure (0.9466) of route estimation at the maximum. Moreover, in the range of the F-measure, the proposed method is $42 \%$ better than incremental map matching, suggesting that the proposed method is less affected by the RSSI threshold. Because the RSSI value varies depending on the remaining battery level and human body or so, the proposed method will be suitable for practical use that it is not easily influenced by the threshold value. On the contrary, the processing time of the proposed method is 493 times slower than that of incremental map matching, and so it is necessary to select a method according to the application.

As future work, we need to develop a method to accelerate the proposed method. In addition, because the proposed method is relatively insensitive to thresholds, it is expected that good results will be obtained in practical use. It is necessary to perform practical evaluation by conducting larger and long-term experiments.

Acknowledgments This research was funded by JSPS Grantin-Aid for Scientific Research 25700009 and SCOPE by Ministry of Internal Affairs and Communications, Japan.

\section{REFERENCES}

[1] Dragan Ahmetovic, Cole Gleason, Chengxiong Ruan, Kris Kitani, Hironobu Takagi, and Chieko Asakawa. 2016. NavCog: a navigational cognitive assistant for the blind, In Proceedings of the 18th International Conference on HumanComputer Interaction with Mobile Devices and Services, 90-99.

[2] Aitor De Blas and Diego López-de-Ipiña. 2017. Improving trilateration for indoors localization using BLE beacons, 2nd International Multidisciplinary Conference on Computer and Energy Science (SpliTech), Split, 1-6.
[3] Sotiris Brakatsoulas, Dieter Pfoser, Randall Salas, and Carola Wenk. 2005. On map-matching vehicle tracking data, In Proceedings of the 31st international conference on Very large data bases, 853-864.

[4] Angelo Chianese, Fiammetta Marulli, Vincenzo Moscato, and Francesco Picciali. 2013. A 'smart' multimedia guide for indoor contextual navigation in Cultural Heritage applications, In Proceedings of the 2013 IEEE International Conference for Indoor Positioning and Indoor Navigation, 1-6.

[5] Pavel Davidson and Robert Piché. 2017. A Survey of Selected Indoor Positioning Methods for Smartphones, IEEE COMMUNICATIONS SURVEYS \& TUTORIALS, VOL. 19, NO. 2, 1347-1370.

[6] Patrick Dickinson, Gregorz Cielniak, Olivier Szymanezyk, and Mike Mannion. 2016. Indoor positioning of shoppers using a network of Bluetooth Low Energy beacons, In Proceedings of the 2016 International Conference on Indoor Positioning and Indoor Navigation, 1-8.

[7] Ramsey Faragher and Robert Harle. 2014. An analysis of the Accuracy of Bluetooth Low Energy for Indoor Positioning Applications, In Proceedings of the 27th International Technical Meeting of the Satellite Division of the Institute of Navigation, 201-210.

[8] Ramsey Faragher and R. Harle. 2015, Location Fingerprinting With Bluetooth Low Energy Beacons, in IEEE Journal on Selected Areas in Communications, vol. 33, no. 11, 2418-2428.

[9] René Hansen, Bent Thomsen, Lone Leth Thomsen, and Filip Stubkjær Adamsen. 2013. SmartCampusAAU - an open platform enabling indoor positioning and navigation, In Proceedings of the 14th IEEE International Conference on Mobile Data Management, vol. 2, 33-38.

[10] Zhiqiang He, Binyue Cui, Wei Zhou, and Shigeki Yokoi. 2015. A proposal of interaction system between visitor and collection in museum hall by iBeacon, In Proceedings of the 10th International Conference on Computer Science and Education, 427-430.

[11] Seigo Ito and Nobuo Kawaguchi. 2005. Bayesian based location estimation system using wireless LAN, In Proceedings of the Third IEEE International Conference on Pervasive Computing and Communications Workshops, 273-278.

[12] Wook Rak Jung and Scott Bell. 2013. Quantitative comparison of indoor positioning on different densities of WiFi arrays in a single environment, In Proceedings of the Fifth ACM SIGSPATIAL International Workshop on Indoor Spatial Awareness, 29-36.

[13] Shinsuke Kajioka, Tomoya Mori, Takahiro Uchiya, Ichi Takumi, and Hiroshi Matsuo. 2014. Experiment of indoor position presumption based on rssi of bluetooth le beacon, In Proceedings of the 2014 IEEE 3rd Global Conference on Consumer Electronics, 337-339.

[14] Shinsuke Kajioka, Daisuke Yamamoto, Takahiro Uchiya, Shoichi Saito, Hiroshi Matsuo, and Ichi Takumi. 2016. Operation of Localization-based Attendance Check System using BLE Beacons, In Proceedings of the Information Processing Society of Japan Technical Reports, 2016-SPT-20, No. 12, 1-7.

[15] Shunsuke Kimura, Takuto Matsumoto, Sakurako Yazawa, Junichi Hoshino, Seunghee Lee, and Masatoshi Hamanaka, 2013. Child Monitoring Service System Using Indoor Location Detection, IPSJ Journal, Vol.12, No.4, 177-183.

[16] Yin Lou, Chengyang Zhang, Yu Zheng, Xing Xie, Wei Wang, and Yan Huang. 2009. Map-matching for lowsampling-rate GPS trajectories, In Proceedings of the ACM SIGSPATIAL 2009, 352-361.

[17] Paul Newson and John Krumm. 2009. Hidden Markov map matching through noise and sparseness, In Proceedings of the ACM SIGSPATIAL 2009, 336-343.

[18] Stange Fraunhofer, Thomas Liebig, Dirk Hecker, Gennady Andrienko, and Natalia Andrienko. 2011. Analytical workflow of monitoring human mobility in big event settings using Bluetooth, in Proceedings of the 3rd ACM SIGSPATIAL International Workshop on Indoor Spatial Awareness, 51-58.

[19] Kenta Urano, Katsuhiko Kaji, Kei Hiroi, and Nobuo Kawaguchi. 2017. A Location Estimation Method using Mobile BLE Tags with Tandem Scanners,In Proceedings of the $2017 \mathrm{ACM}$ International Joint Conference on Pervasive and Ubiquitous Computing, 577-586.

[20] Christopher E. White, David Bernstein, and Alain L. Kornhauser. 2000. Some map matching algorithms for personal navigation assistants, Transportation Research C, Vol. 8, 91-108.

[21] Moustafa Youssef. 2015. Towards truly ubiquitous indoor localization on a worldwide scale, In Proceedings of the 23rd SIGSPATIAL International Conference on Advances in Geographic Information Systems, Article No.12.

[22] Ahmed Shokry, Moustafa Elhamshary, and Moustafa Youssef. 2017. The Tale of Two Localization Technologies: Enabling Accurate Low-Overhead WiFi-based Localization for Low-end Phones, In Proceedings of the 25th ACM SIGSPATIAL International Conference on Advances in Geographic Information Systems, Article No. 42.

[23] Faheem Zafari and Ioannis Papapanagiotou, 2015. Enhancing iBeacon Based Micro-Location with Particle Filtering, In Proceedings of the 2015 IEEE Global Communications Conference, $1-7$. 